\title{
LicA induces autophagy through ULK1/Atg13 and ROS pathway in human hepatocellular carcinoma cells
}

\author{
QIANG NIU*, WEI ZHAO*, JIN WANG, CHUNMING LI, TAO YAN, WEI LV, GUOJING WANG, \\ WEIHONG DUAN, TAO ZHANG, KUNNAN WANG and DINGHUAZHOU
}

Department of Hepatobiliary Surgery, Rocket Army General Hospital, Beijing 100088, P.R. China

Received December 16, 2016; Accepted February 7, 2018

DOI: $10.3892 / \mathrm{ijmm} .2018 .3499$

\begin{abstract}
Chemotherapy is the best choice for the vast majority of hepatocellular carcinoma patients at late stage, but few effective chemotherapy drugs are available in clinic. Licochalcone A (LicA) is a new chemotherapy drug inducing apoptosis as Bcl-2 inhibitor, but few studies report on LicA-induced autophagy. This study investigated the phenomenon and mechanisms of LicA-induced autophagy looking for a targeted combination drug. Human hepatocellular carcinoma cells (HCCs) were treated with LicA, to detect markers of autophagy and to investigate the mechanisms. In order to investigate the role of reactive oxygen species (ROS) in LicA-induced autophagy, ROS, glutathione (GSH) and $\mathrm{O}_{2}{ }^{-}$were measured in LicA treated HCCs, and antioxidant $\mathrm{N}$-Acetyl-L-cysteine (NAC) was cotreated with LicA in HCCs, then mechanisms of ROS-induced autophagy was investigated in LicA or LicA combined with NAC treated HCCs. Finally, the LicA-induced apoptosis was detected in LicA combined with NAC treated HCCs. We first report that LicA can induce autophagy through ULK1/Atg13 and ROS pathway in HCCs, suppression of LicA-induced ROS through antioxidant NAC can enhance LicA-induced apoptosis, promoting the function of LicA killing HCCs. LicA can activate the ULK1/Atg13 complex which is upstream of autophagy, additionally, LicA also can promote ROS generation, ROS trigger the expression level of TSC1/2 complex, PRAS40, CTMP, PP2A, PDK1 and
\end{abstract}

Correspondence to: Dr Dinghua Zhou, Department of Hepatobiliary Surgery, Rocket Army General Hospital, 16 Xinjie Kouwai Street, Xicheng, Beijing 100088, P.R. China

E-mail: zhoudh@sina.com

*Contributed equally

Abbreviations: LicA, licochalcone A; ROS, reactive oxygen species; NAC, N-Acetyl-L-cysteine; HCCs, human hepatocellular carcinoma cells; GSH, glutathione; CTMP, carboxy-terminal modulator protein; $\mathrm{PKB}$, protein kinase $\mathrm{B}$; $\mathrm{PP} 2 \mathrm{~A}$, protein phosphatase $2 \mathrm{~A}$; TSC, tuberous sclerosis complex

Key words: licochalcone A, reactive oxygen species, autophagy, hepatocellular carcinoma cells
Rubicon change, these molecules are upstream of autophagy. In conclusion, LicA can induce autophagy through ULK1/ Atg13 and ROS pathway in HCCs, LicA combined with NAC can enhance LicA-induced apoptosis. Our results may provide a novel design for clinical hepatocellular carcinoma therapy trials.

\section{Introduction}

Primary liver cancer is the main diagnosis of hepatocellular carcinoma cell (HCC). The mortality of HCC is ranked second in cancer-related death worldwide, there are 695,900 death and 748,300 new cases every year $(1,2)$. Although the 5 -year survival rate is $>70 \%$ when the patients are diagnosed as early stage and have good prognosis, The vast majority of patients are diagnosed as late stage, and the 5-year survival rate is $<16 \%$ (1). The treatment of HCC includes surgery and adjuvant chemoradiation (1). Due to the limitation of surgical therapy with tumor size, intra-hepatic metastases and hepatic functional reserve sufficiency, few patients are suitable to receive this treatment (3). Chemotherapy is the final choice of the vast majority of patients, however, the HCC appear moderately tolerated, reducing the effect of chemotherapy $(1,3)$. Therefore, exploring the mechanisms of chemotherapy agents that are tolerated and to select the appropriate adjuvant chemotherapy is very important.

Licochalcone A (LicA) is a chalcone constituent of licorice, it has anti-inflammatory, antitumor and anti-bacterial ability (4). LicA is a potent inhibitor of Bcl-2 protein expression which is the anti-apoptotic proteins in various tumors, LicA also can induce apoptosis in several cancer cell lines contributing to the antitumor effect $(5,6)$. In human HCCs, the migration and invasion can be suppressed by LicA. LicA has anti-inflammatory ability through suppressing nuclear factor $-\kappa \mathrm{B}(\mathrm{NF}-\kappa \mathrm{B})$ and activator protein-1 (AP-1), and tumor angiogenesis is closely linked to inflammation, LicA can also inhibit tumor angiogenesis $(7,8)$. These results suggest that LicA may have potential as antitumor therapy.

Autophagy is a protective mechanism, many chemotherapy drugs can induce autophagy, and reduce the effect of chemotherapy (9). Few reports exist on LicA inducing autophagy in HCCs, in this study, LicA induced autophagy in HCCs through activating ULK1/Atg13 complex and reactive oxygen species (ROS), ULK1/Atg13 complex is upstream of 
autophagy, and ROS was upstream of autophagy-related molecule. Moreover, the antioxidant N-Acetyl-L-cysteine (NAC) can inhibit LicA-induced autophagy through suppressing ROS in HCCs, promoting apoptosis and enhancing cell death rate, suggesting LicA may be a good chemotherapy drugs for HCC, especially as co-treatment with an antioxidant.

\section{Materials and methods}

Cell culture. Human HCCs HuH7 and HepG2 were purchased from American Type Culture Collection (ATCC, Manassas, VA, USA). The cells were cultured in DMEM conditioned medium, including $2 \mathrm{mM}$ L-glutamine, $100 \mathrm{U} / \mathrm{ml}$ penicillin, $100 \mathrm{mg} / \mathrm{ml}$ streptomycin and $10 \%$ fetal bovine serum (FBS), in the normal culture environment with $37^{\circ} \mathrm{C}$ and $5 \% \mathrm{CO}_{2}$, and all the cell culture regents were purchased from Gibco Laboratories (Grand Island, NE, USA).

Western blot analysis. For each sample, all the cells were lysed for $30 \mathrm{~min}$ in lyses buffer (Beyotime, Bejing, China) on ice, and the cell debris was centrifuged at $12,000 \mathrm{x}$ g for $12 \mathrm{~min}$ at $4^{\circ} \mathrm{C}$, protein concentration were detected by BCA (Beyotime), using 8-15\% sodium dodecyl sulfate-polyacrylamide gel electrophoresis (SDS-PAGE) to separate the proteins with $12 \mathrm{~V}$, the separated proteins were blotted onto PVDF membrane, blocking $1 \mathrm{~h}$ at room temperature with $5 \%$ nonfat drymilk in $0.05 \%$ Tween-20 in PBS (PBST), incubating the antibody for $24 \mathrm{~h}$ at $4^{\circ} \mathrm{C}$ with 1:1,000 concentration, washing PVDF membrane 3 times for $10 \mathrm{~min}$ in PBST, incubating secondary antibody at $37^{\circ} \mathrm{C}$ for $1 \mathrm{~h}$ with the $1: 10,000$ concentration, washing PVDF membrane 3 times for $10 \mathrm{~min}$ in PBST. All the antibodies were purchased from Santa Cruz Biotechnology, Inc. (Santa Cruz, CA, USA).

Acridine orange $(A O)$ staining. Cells were treated with drugs, slightly washed 3 times with PBS, using 4\% faraformaldehyde to fix for $10 \mathrm{~min}$, stained with $1 \mathrm{mg} / \mathrm{l} \mathrm{AO}$ dye liquor for $20 \mathrm{~min}$ at $37^{\circ} \mathrm{C}$ in the dark, and then observed under fluorescence microscope (Nikon TE2000; Nikon, Tokyo, Japan).

GFP-LC3 transfection. HuH7 and HepG2 cells were transfected with GFP-LC3 vector (GeneChem, Shanghai, China) through Lipofectamine 2000 (Invitrogen Life Technology, Carlsbad, CA, USA). After 18 h, cells were treated with drugs for $24 \mathrm{~h}$, using $4 \%$ faraformaldehyde to fix for $10 \mathrm{~min}$, washed 3 times with PBS, and then observed under fluorescence microscope (Nikon TE2000; Nikon).

Co-immunoprecipitation experiments. HepG2 cells were treated with drugs for $12 \mathrm{~h}$, lysing cells in hypotonic lyses buffer (Beyotime) on ice for $30 \mathrm{~min}$, collecting the supernatant to pellet nuclei in whole-cell lysate at a low speed $(1,000 \mathrm{x} \mathrm{g})$, preclearing whole-cell lysate with protein A-agarose, adding ULK 1 or Atg13 antibodies for $1 \mathrm{~h}$ at $4^{\circ} \mathrm{C}$. Then the immunoprecipitates were captured on protein A-agarose and detected by immunoblotting with ULK1 or Atg13 antibodies, respectively.

Cell viability assay. Cell viability was detected by cell counting kit-8 (CCK-8) agent. Cells on a 96-well plate were left to attach overnight, then treated with drugs for $24 \mathrm{~h}$.
Medium was removed the and washed 3 times with PBS, then $90 \mu \mathrm{l}$ DMEM and $10 \mu \mathrm{l}$ CCK-8 was added into each well, and incubated for $1.5 \mathrm{~h}$ at $37^{\circ} \mathrm{C}$ in the dark. OD values were measured by microplate reader at $450 \mathrm{~nm}$.

ROS detection. ROS level was measured by 2,7-dichlorofluorescein diacetate (DCFH-DA) (Beyotime). Cells on a 96-well plate were let to attached overnight, then treated with drugs for $24 \mathrm{~h}$. The medium was removed and washed 3 times with PBS, then incubated with DCFH-DA at $37^{\circ} \mathrm{C}$ for $30 \mathrm{~min}$, measuring the ROS levels through fluorescence microplate reader with $488 \mathrm{~nm}$ excitation wavelength and $525 \mathrm{~nm}$ emission wavelength.

Cell death assay. Cell death ratio was detected by trypan blue. The cells were treated with drugs, and then digested adding $0.4 \%(\mathrm{w} / \mathrm{v})$ trypan blue solution into cell suspension at volume ratio 1:9. Counting the dead cells and total cells with a microscope, the dead cells cannot exclude the dye, total death rate $=($ the number of dead cell/total cell $) \times 100 \%$.

Transmission electron microscopy (TEM). Samples were processed with the standard protocol for TEM. TEM was performed on a JEOL 1230 TEM at an accelerating voltage of $80 \mathrm{kV}$. Images were acquired with an AMT Advantage Plus $2 \mathrm{~K} \times 2 \mathrm{~K}$ digital camera connected to the TEM.

Transfection experiments. siRNA transient transfections were performed with Lipofectamine 2000 (Invitrogen Life Technology) according to the manufacturer's protocol. After $36 \mathrm{~h}$ transfection, drugs were added, and after an additional $24 \mathrm{~h}$ the cells were collected for western blot analysis.

Apoptosis detection. Apoptosis was detected by FACScan flow cytometer. Treating the cell with drugs, cells were incubated with Annexin V-FITC and propidium-iodide (PI) (both from Beyotime) at room temperature for $10 \mathrm{~min}$. FACScan flow cytometer was used to analyses the apoptosis ratio.

Statistic analysis. The data are represented as the mean \pm SD from triplicate experiments. Two-way ANOVA was used to analyze the variance of different groups. A threshold of $\mathrm{P}<0.05$ was defined as statistically significant.

\section{Results and Discussion}

When autophagy occurs, the cytosolic LC3 will be cleaved to short peptide hydrolysis LC3 II which is located on the membrane of autophagosome, therefore, LC3 II is the marker of autophagy that can reflect the level of autophagy. Because the lower $\mathrm{PH}$ value around autophagosome, AO can penetrate into acidic organelles and appear as red fluorescence, staining with $\mathrm{AO}$, and the red fluorescence intensity also can reflect the level of autophagy. Moreover, TEM is the gold standard of autophagosome. LicA can significant enhance the expression of LC3 II, the induced ability was time-dependent and dose-dependent, and the best dose was $50 \mu \mathrm{M}$, the best time was $24 \mathrm{~h}$ (Fig. 1A and B). Compared to the positive control rapamycin treatment, the level of induced-LC3 II has no significant difference, when LicA is co-treated with chlo- 

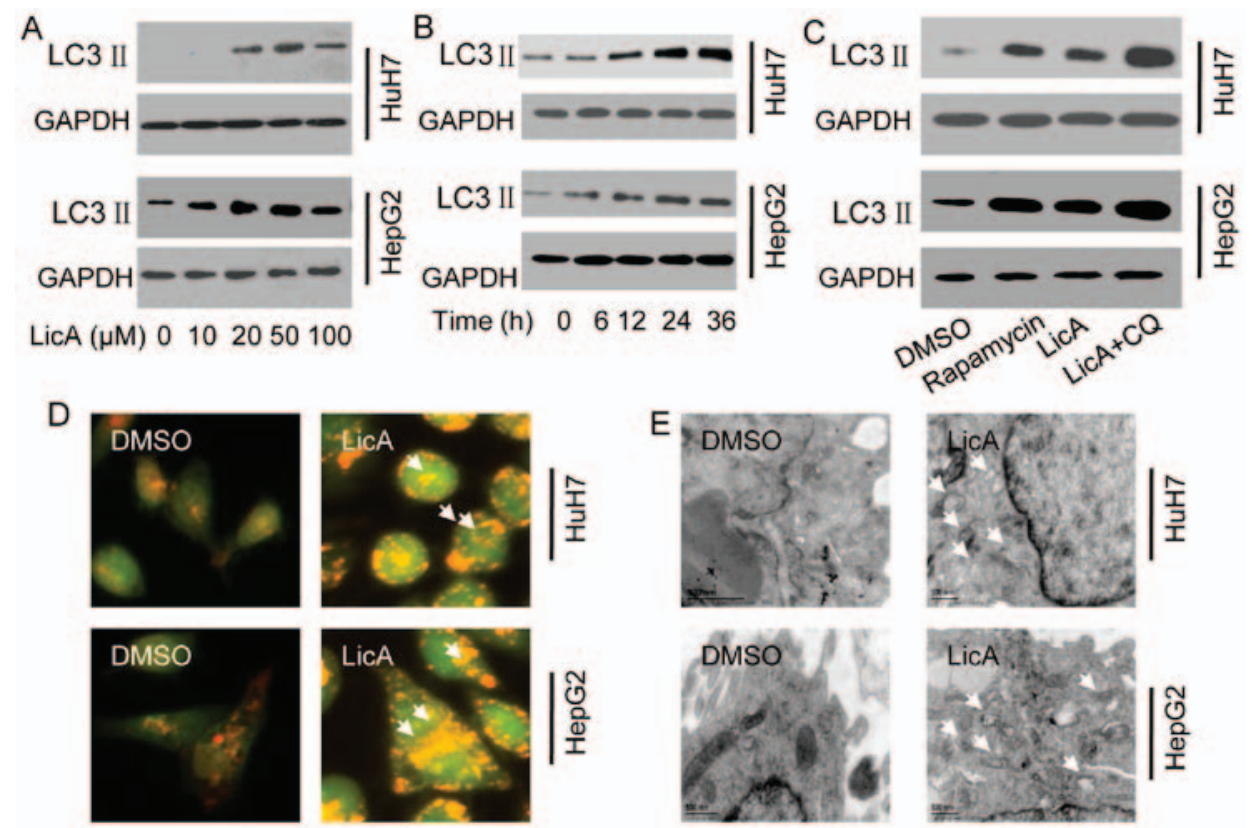

Figure 1. Licochalcone A (LicA) induces autophagy in hepatocellular carcinoma cells (HCCs). (A) HCCs were treated with different doses of LicA for $24 \mathrm{~h}$. (B) HCCs were treated with $50 \mu \mathrm{M}$ LicA for different times. (C) HCCs were treated with $50 \mu \mathrm{M}$ LicA, $50 \mu \mathrm{M}$ LicA combined with $40 \mu \mathrm{M}$ chloroquine (CQ) or $50 \mu \mathrm{M}$ rapamycin for $24 \mathrm{~h}$. (D) HCCs were treated with $50 \mu \mathrm{M} \mathrm{LicA}$ for $24 \mathrm{~h}$ and processed acridine orange (AO) staining (E) and transmission electron microscopy (TEM) (arrows) were examined to evaluate the level of LicA-induced-autophagy.

roquine (CQ) which can block the lysosome and engulf the autophagosome and contribute to LC3-II accumulation, the level of induced-LC3 II was significantly increased (Fig. 1C). AO staining show that the red fluorescence intensity of cells which were treated with $50 \mu \mathrm{M}$ LicA was significant higher than the cells which were treated with $0.1 \%$ dimethyl sulfoxide (DMSO) (Fig. 1D). The TEM results also prove that the number of autophagosome in the cells which were treated with $50 \mu \mathrm{M}$ LicA was significant higher than the cells which were treated with $0.1 \%$ DMSO (Fig. 1E). The above results means that LicA can significantly induce autophagy in HCCs.

ICo-immunoprecipitation (Co-IP) pull-down assay was used to investigate the mechanism of LicA-inducedautophagy. HepG2 cells were treated with $50 \mu \mathrm{M}$ LicA or not, immunoprecipitation of ULK1 can pull down Atg13, and immunoprecipitation of Atg13 also can pull down ULK1, that means ULK1 and Atg13 were bound to each other forming a complex in HCCs, and LicA can activate the complex which is upstream of autophagy (Fig. 2A and B). Knocking down ULK1 by ULK1-siRNA in HCCs, the level of LicA-induced LC3 II was significant lower than co-siRNA, but still higher than DMSO treatment (Fig. 2C), similarly, staining with $\mathrm{AO}$, the red fluorescence intensity was significant lower than co-siRNA, but still higher than DMSO treatment (Fig. 2D), thus ULK1/Atg13 complex is one of the regulating molecules of LicA-induced autophagy, not the unique regulating molecule.

ROS is one of the survival and proliferation regulator factors of cancer cells, and also regulate autophagy. LicA can suppress the glutathione (GSH) generation (Fig. 3A) and promote the $\mathrm{O}_{2}^{-}$generation (Fig. 3B), finally contributing to ROS generation (Fig. 3C and D) in HCCs. Moreover, when HCCs were cotreated with LicA and antioxidant NAC, the level of GSH, $\mathrm{O}_{2}^{-}$and ROS reversed partly (Fig. 3A-D). These results show that LicA can promote ROS generation in HCCs, and the NAC can inhibit the phenomena.

The above results have proved that LicA can promote ROS generation and NAC can reverse the effect of LicA in HCCs. When HCCs were cotreated with LicA and NAC, the level of LicA-induced LC3 II was lower than HCCs treated with only LicA, but also higher than DMSO treatment (Fig. 4A). At the same time the quantity of LC3-GFP puncta and the red fluorescence intensity with AO staining in HCCs was cotreated with LicA and NAC were lower than those treated with LicA alone, but also higher than DMSO treatment (Fig. 4B and C). These results indicated that LicA can induce ROS-related autophagy, and NAC can inhibit the same. Previous studies have reported that ROS was upstream of many molecules, and ROS was not the direct inducing factor of autophagy. In order to investigate the downstream ROS pro-autophagy pathways, other regulated factors were detected in HCCs. Sarbassov et al (10) have reported that TSC1/2 complex was upstream of mTORC1, and mTORC1 was a universal regulator of autophagy (11). So TSC1/2 complex was detected in this work, the results show that TSC1/2 complex can be activated by LicA through ROS pathway, and can be inhibited by NAC that can suppress ROS generation (Fig. 5). PRAS40 is a metabolism protein bound to mTOR complex, and acts as the receptor of mTOR, PRAS40 also can regulate the proliferation in various cell lines $(12,13)$. The results of this work indicated that PRAS40 can be regulated by LicAinduced-ROS in HCCs, and when HCCs were cotreated with LicA and NAC, the change of PRAS40 expression level was inhibited (Fig. 5). Carboxy-terminal modulator protein (CTMP), as an endogenous inhibitor of Akt, also can influence the phosphoinositide 3-kinase (PI3K) pathway through activating protein kinase $\mathrm{B}$ (PKB) signaling, leading to insulin signaling regulation (14). These studies support our 

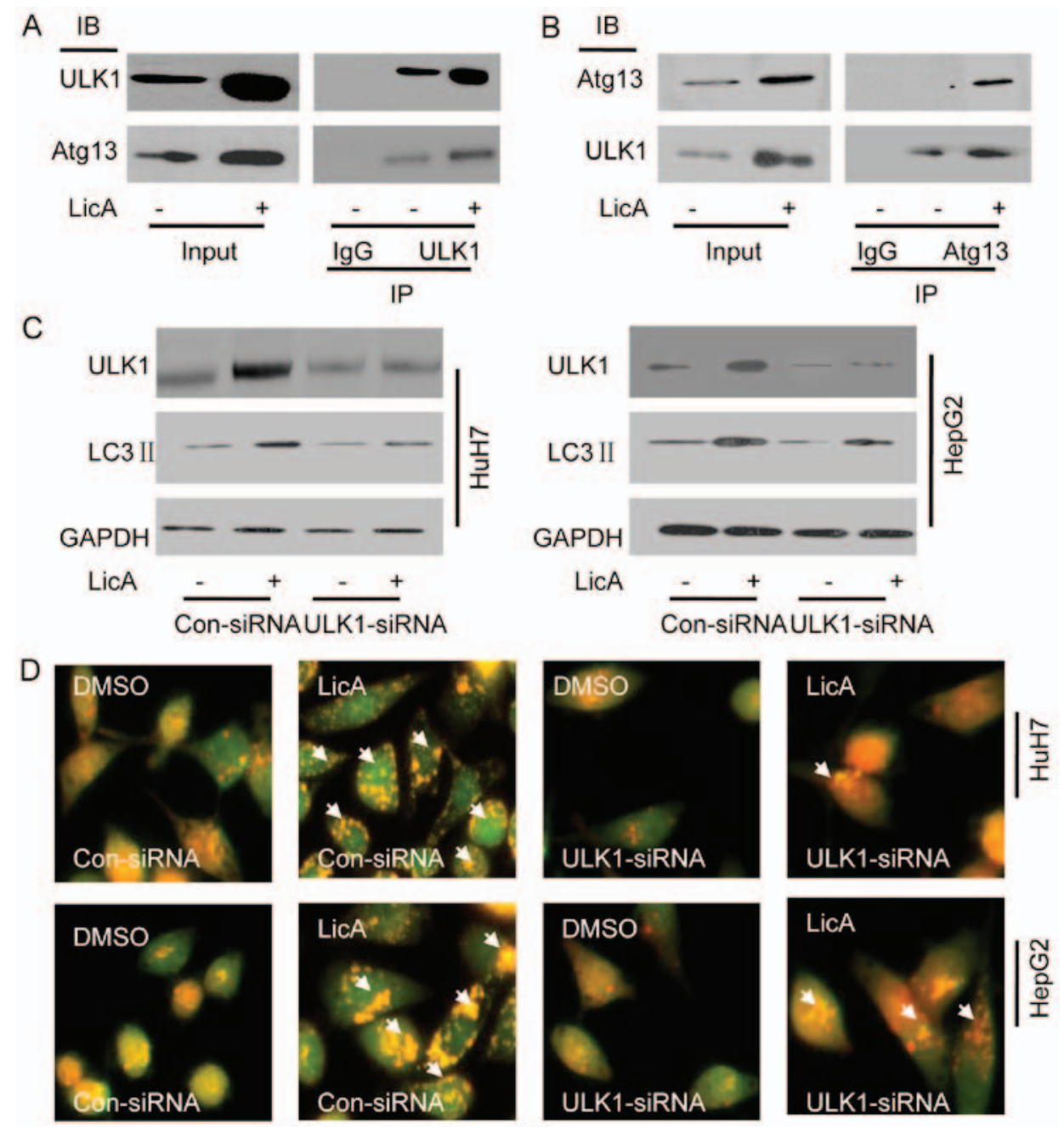

Figure 2. Licochalcone A (LicA) activates ULK1/Atg13 signal pathway. (A and B) HepG2 cells were treated with $50 \mu \mathrm{M}$ LicA for $12 \mathrm{~h}$. (C) Hepatocellular carcinoma cells (HCCs) were transfected with Con-siRNA or ULK1-siRNA, $36 \mathrm{~h}$ later, treated with $0.1 \%$ DMSO or $50 \mu \mathrm{M}$ LicA for $24 \mathrm{~h}$, and then possessing the immunoblot or (D) acridine orange (AO) staining.

results that CTMP can regulate autophagy, moreover, CTMP was downstream in LicA-induced autophagy via promoting ROS generation in HCCs, similarly, the phenomenon can be changed by NAC (Fig. 5). Protein phosphatase 2A (PP2A) is a key regulator of cell cycle, also the target for anticancer drugs, PP2A can regulate the autophagy through AMPK phosphorylation. These studies showed PP2A may be an important regulator in LicA-induced autophagy $(15,16)$ the results in our study proved the hypothesis as PP2A acted as the regulator of LicA-induced autophagy through promoting ROS generation, and ROS inhibitor NAC can block this progress (Fig. 5). PDK1 is an AGC kinase of the PKB family which contain a $\mathrm{PH}$ domain regulating cell proliferation, apoptosis and differentiation, PDK1 is upstream of Akt, and impair the autophagic effect through targeting activated c-Src (p-Src) $(17,18)$. LicA can inhibit the PDK1 expression through ROS in LicAinduced autophagy, and NAC reversed this (Fig. 5). Rubicon is a subunit of PI3KC3, containing cysteine-rich protein, acting as a regulator of Beclin1-UVRAG-Vps34 complex to regulate autophagy (19). LicA-induced ROS can inhibit Rubicon expression in LicA-induced autophagy, and NAC is the inhibitor of this program (Fig. 5). These results indicated that ROS is the pathway of LicA-induced autophagy in HCCs.
LicA can induce autophagy in HCCs through promoting ROS generation, and autophagy is a protective mechanism for cells, the antioxidant NAC can inhibit ROS generation, moreover, LicA is the activator of apoptosis in various cancer cells. Therefore, in order to investigate the function of ROS-mediated autophagy in LicA-induced apoptosis for HCCs, HCCs were cotreated with LicA and NAC or treated with LicA alone, the cell death ratio and cell viability ratio were detected, LicA can induced cell death and decrease cell viability in HCCs, and NAC can enhance the effect of LicA in killing HCCs (Fig. 6A and B). The apoptosis marker caspase-3 was detected, LicA can promote caspase-3 expression and NAC enhance the effect, which means that NAC could enhance LicA-induced apoptotic in HCCs (Fig. 6C). Annexin V-FITC and PI results indicated that LicA NAC enhanced the LicA-induced cell apoptosis ratio (Fig. 6D). Thus, the above results indicated that suppression of ROS-mediated autophagy induced by LicA enhanced LicAinduced apoptosis.

In this study, we first report that LicA can induce autophagy through ULK1/Atg13 and ROS pathway in HCCs, suppression of LicA-induced ROS through antioxidant NAC can enhance LicA-induced apoptosis, promoting the function 

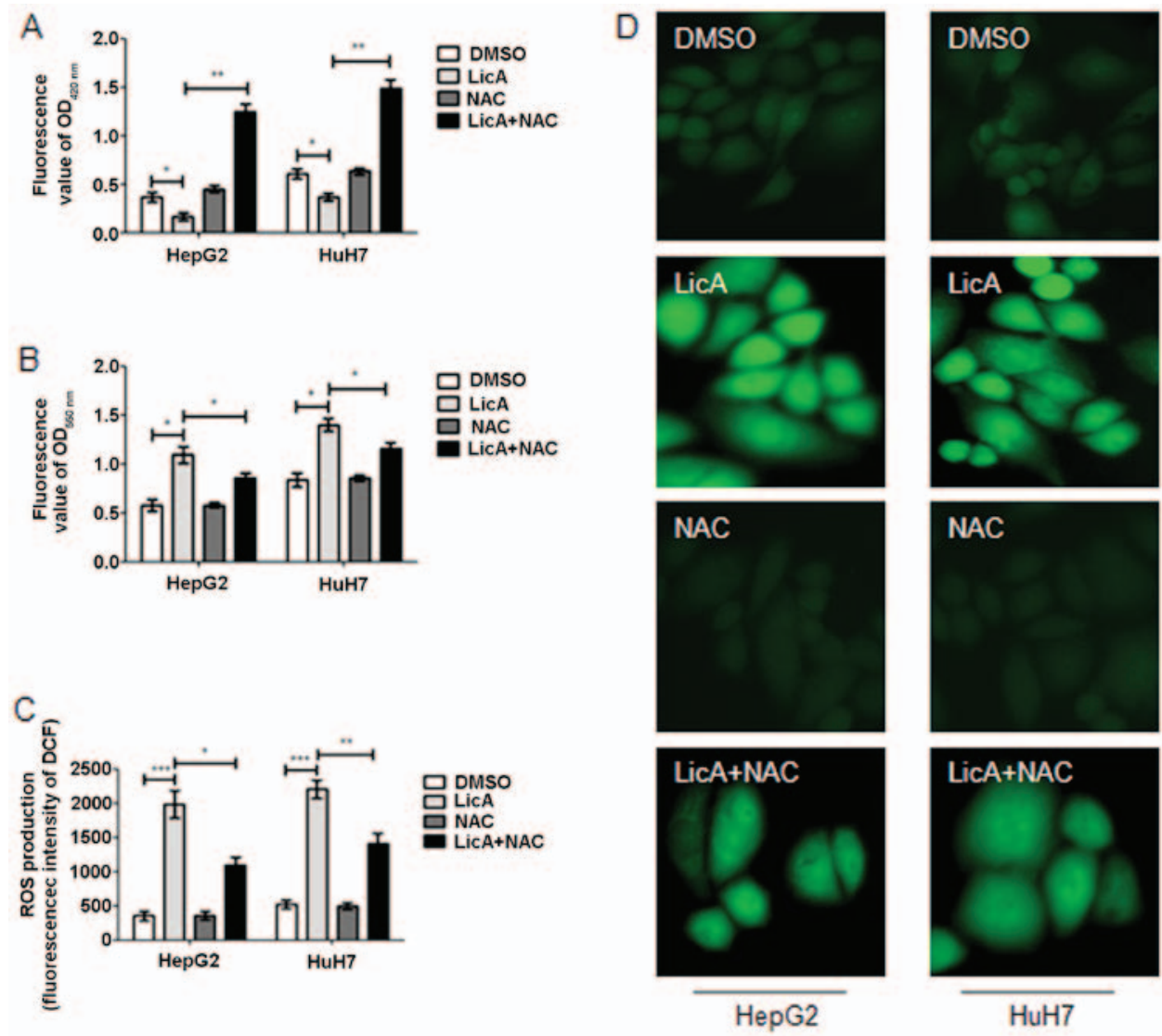

Figure 3. Licochalcone A (LicA) promotes reactive oxygen species (ROS) generation in hepatocellular carcinoma cells (HCCs). HCCs were pretreated with antioxidant N-Acetyl-L-cysteine (NAC) for $2 \mathrm{~h}$, and treated with $0.1 \%$ DMSO or $50 \mu \mathrm{M} \mathrm{LicA}$ for $24 \mathrm{~h}$. (A) Measuring the level of glutathione (GSH) by ELISA kit at $420 \mathrm{~nm}$. (B) The level of $\mathrm{O}_{2}^{-}$was measured by ELISA kit at $550 \mathrm{~nm}$. The level of ROS was measured by 2,7-dichlorofluorescein diacetate (DCFH-DA) using $(C)$ fluorescence microplate reader or (D) fluorescence microscope. ${ }^{*} \mathrm{P}<0.05,{ }^{* *} \mathrm{P}<0.01$ and ${ }^{* * * *} \mathrm{P}<0.005$.
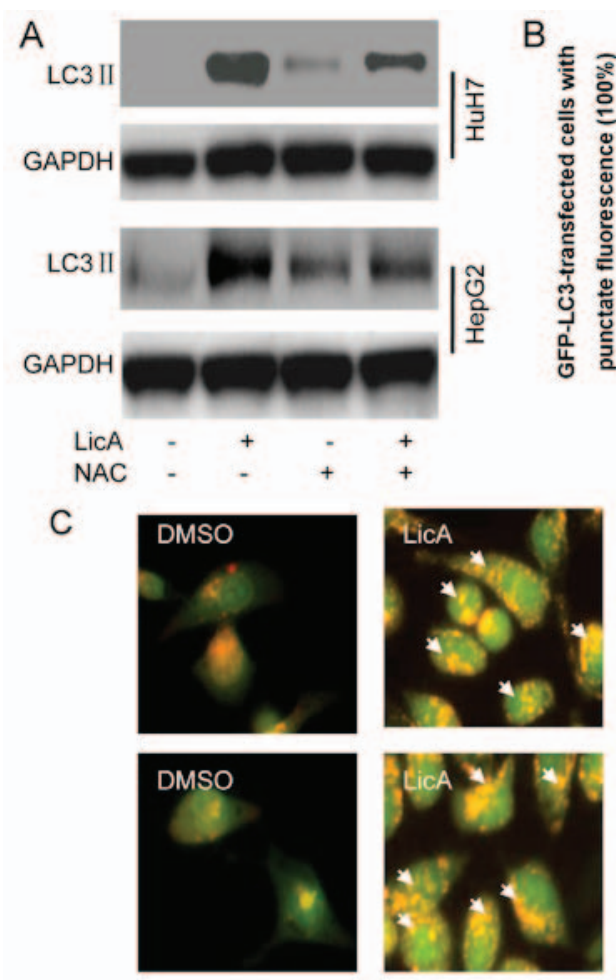
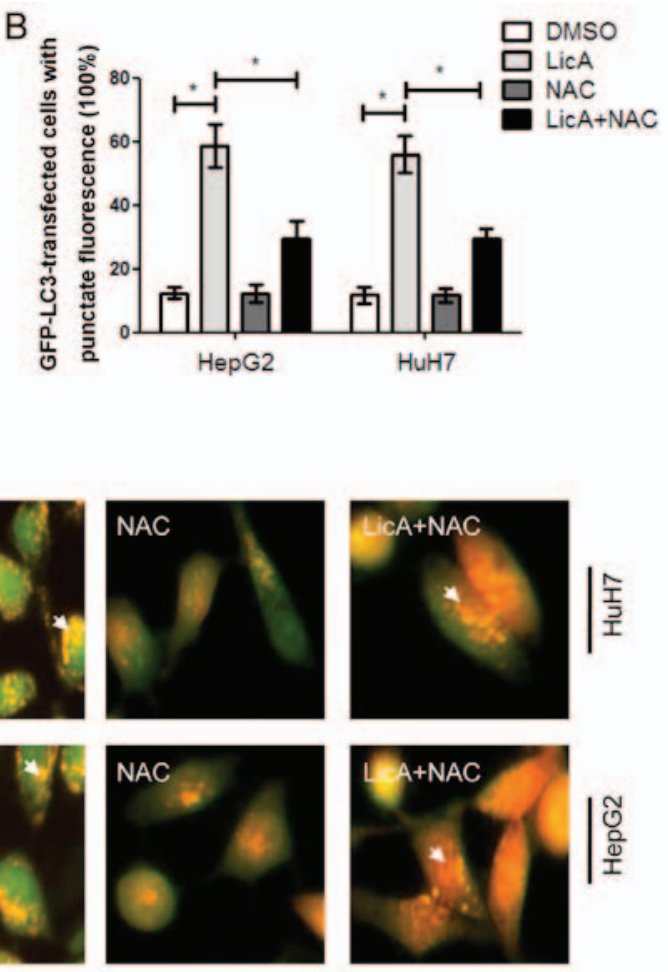

Figure 4. Licochalcone A (LicA) induces autophagy through reactive oxygen species (ROS) pathway in hepatocellular carcinoma cells (HCCs). HCCs were pretreated with antioxidant N-Acetyl-L-cysteine (NAC) for $2 \mathrm{~h}$, and treated with $0.1 \%$ DMSO or $50 \mu \mathrm{M}$ LicA for $24 \mathrm{~h}$. (A) The expression level of LC3 was detected by immunoblot. (B) HCCs were transfected with GFP-LC3 plasmid, and counting the LC3-GFP puncta positive cells (at least 5 puncta) with fluorescence microscope. (C) acridine orange (AO) staining for detecting autolysosomes. ${ }^{*} \mathrm{P}<0.05$. 

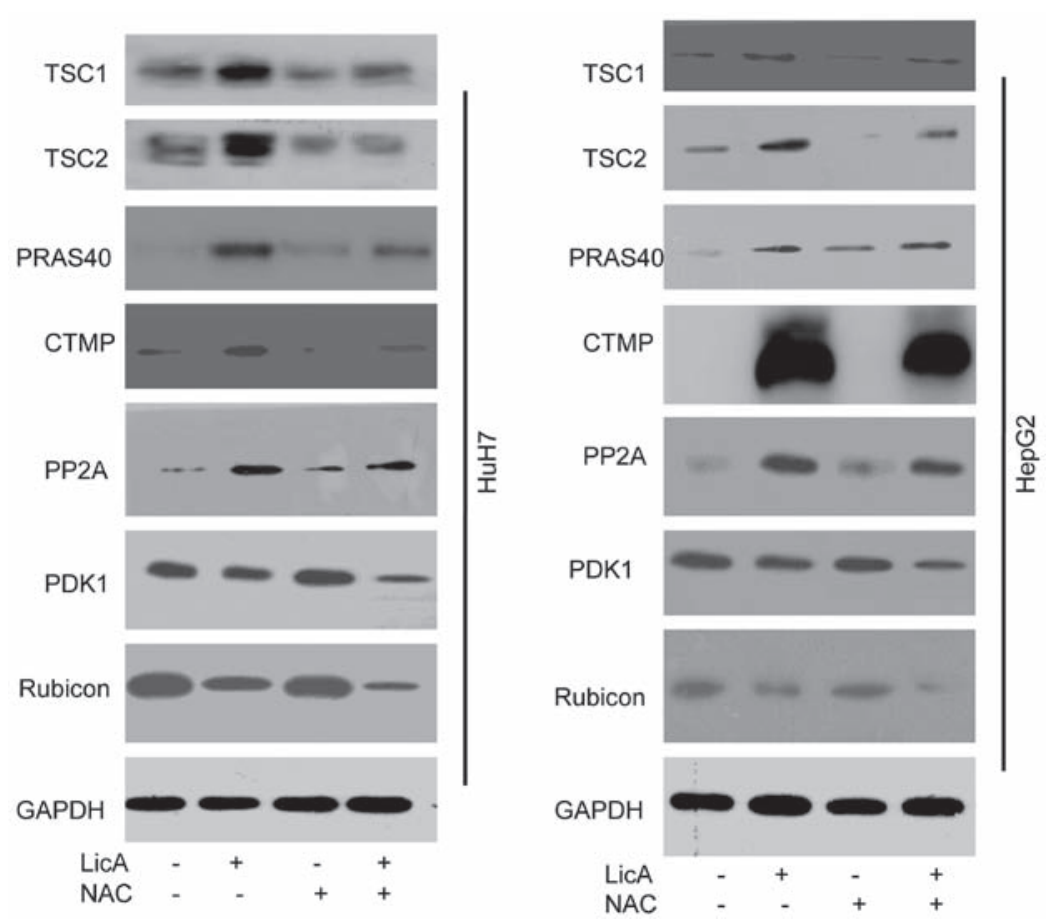

Figure 5. Reactive oxygen species (ROS) has an important role in licochalcone A (LicA)-induced autophagy. Hepatocellular carcinoma cells (HCCs) were pretreated with antioxidant N-Acetyl-L-cysteine (NAC) for $2 \mathrm{~h}$, and treated with $0.1 \%$ DMSO or $50 \mu \mathrm{M}$ LicA for $24 \mathrm{~h}$, the expression of TSC1/2 complex, PRAS40, carboxy-terminal modulator protein (CTMP), protein phosphatase 2A (PP2A), PDK1 and Rubicon were measured by immunoblotting.
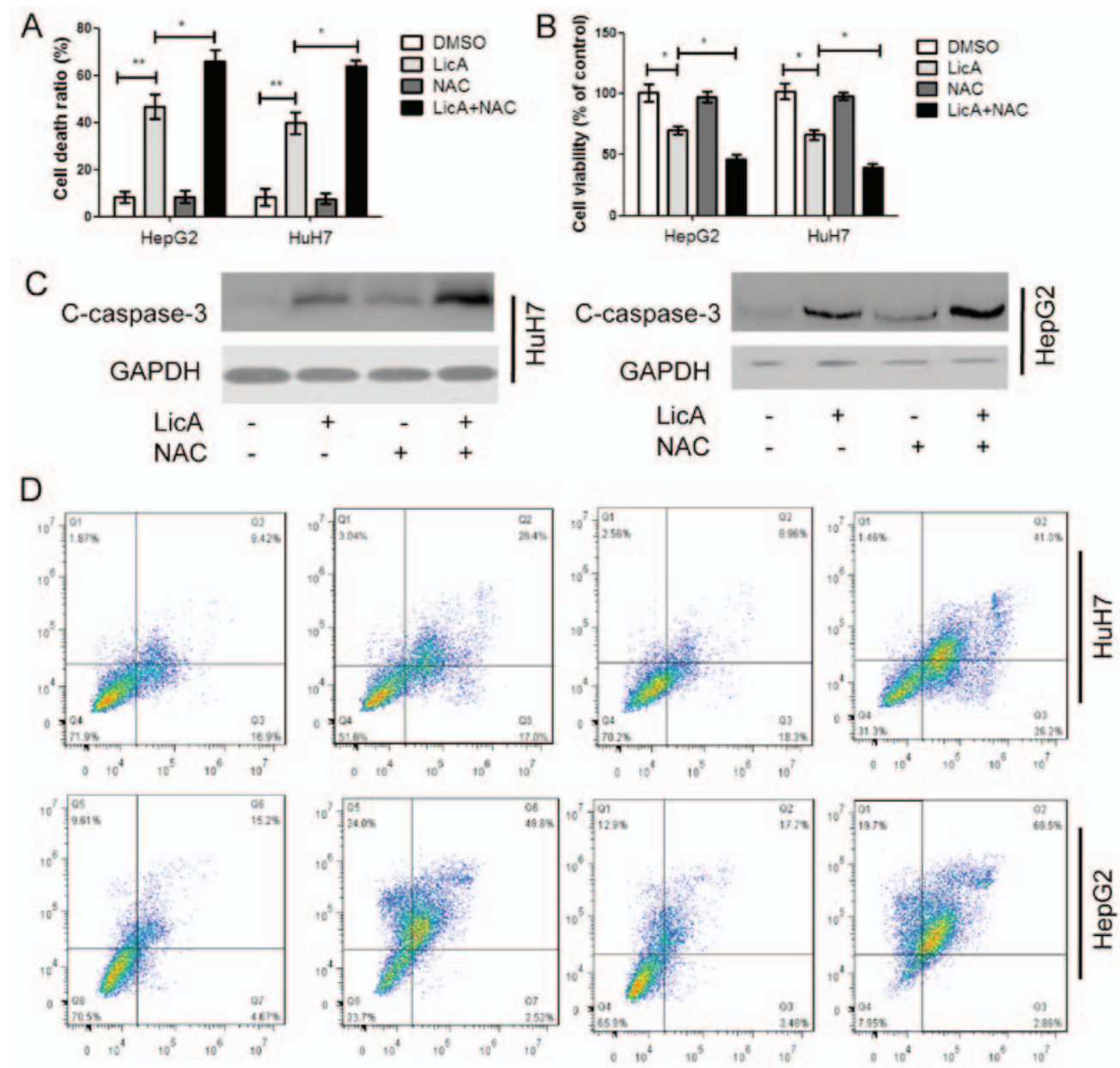

Figure 6. Antioxidant N-Acetyl-L-cysteine (NAC) enhances LicA-induced apoptosis. Hepatocellular carcinoma cells were pretreated with antioxidant NAC for $2 \mathrm{~h}$, and treated with $0.1 \%$ DMSO or $50 \mu \mathrm{M} \mathrm{LicA}$ for $24 \mathrm{~h}$. (A) Cell death ratio was measured by Trypan blue staining. (B) Cell viability was measured by cell counting kit-8 (CCK-8) kit. (C) Caspase-3 was measured by immunoblotting. (D) Apoptosis was detected by FACScan flow cytometer. ${ }^{*} \mathrm{P}<0.05$ and ${ }^{* *} \mathrm{P}<0.01$. 


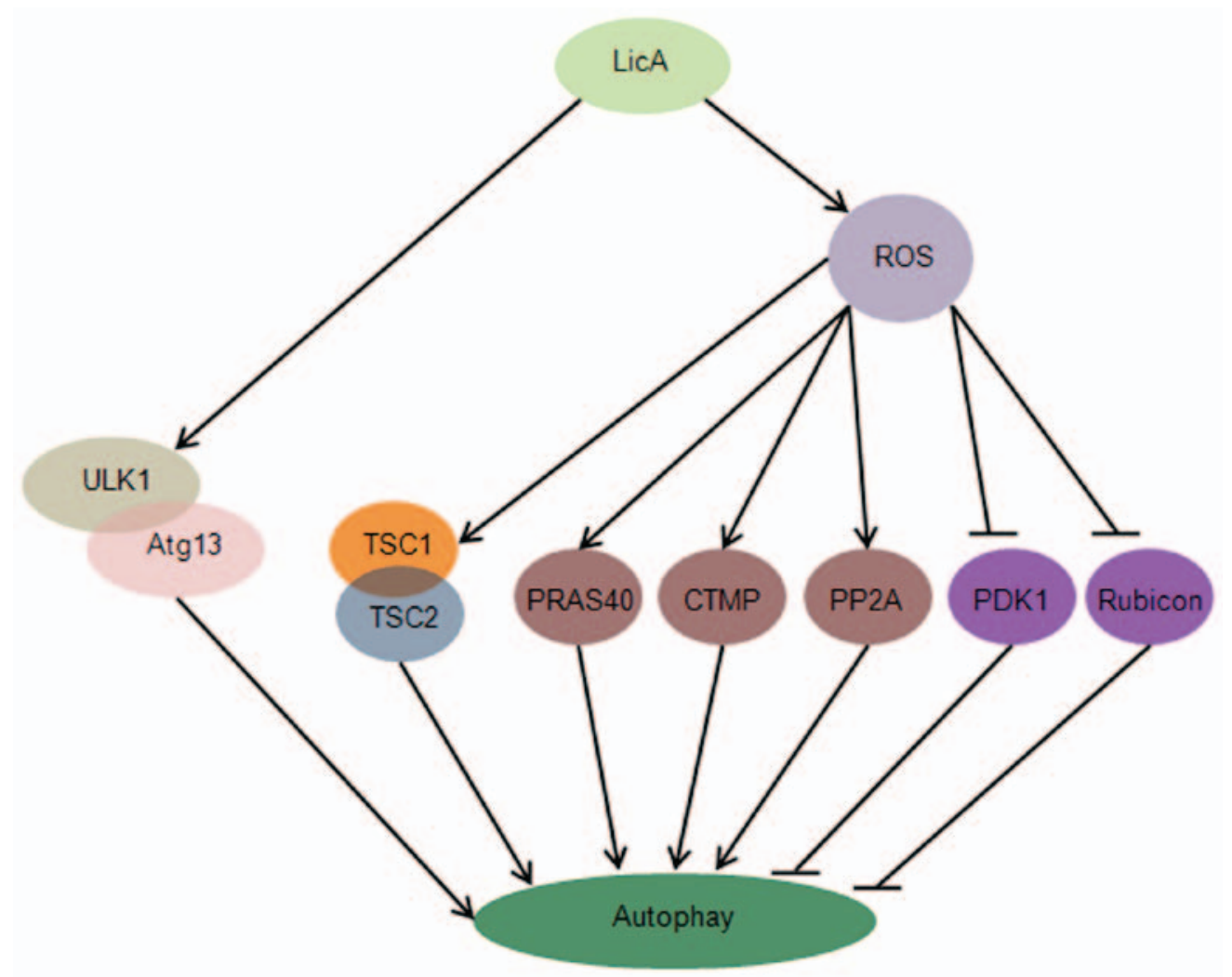

Figure 7. The pathway of licochalcone A (LicA)-induced autophagy. LicA activates the ULK1/Atg13 complex, and LicA promotes reactive oxygen species (ROS) generation, following TSC1/2 complex, PRAS40, carboxy-terminal modulator protein (CTMP), protein phosphatase 2A (PP2A), PDK1 and Rubicon changed triggering of ROS-mediated autophagy.

of LicA killing HCCs. LicA can activate the ULK1/Atg13 complex which is upstream of autophagy, LicA can promote ROS generation, ROS trigger the expression level of TSC1/2 complex, PRAS40, CTMP, PP2A, PDK1 and Rubicon change, these molecules are upstream of autophagy (Fig. 7).

LicA has been reported to have antitumor, anti-angiogenesis and anti-inflammatory ability, and considered as Bcl-2 inhibitor $(4,5,6,20)$. LicA can block cell cycle progression through regulating extracellular signal-regulated kinase 1/2 (ERK1/2) and $\mathrm{Rb}$ phosphorylation (21). Animal experiments show that, LicA significantly inhibits tumor growth, and reduces the cell nuclear antigen, cyclooxygenase-2 (COX-2) and inducible nitric oxide synthase (iNOS), contributing to survival. Moreover, previously it has been reported that LicA can inhibit matrix metalloproteinase-9 (MMP-9) and vascular endothelial growth factor receptor-2 (VEGFR-2) expression in various cancer cells, indicating that LicA can mediate metabolism and angiogenesis $(8,20)$.

Autophagy is a double membrane structure autophagosome formed in the no ribosome attachment region of reticulum membrane, engulfing the waste proteins or aged organelles, fused with lysosomes and forming autolysosome, degradation its contents. Therefore, autophagy is necessary for cell metabolism, and contributes to cell survival. LicA is the Bcl-2 inhibitor, and $\mathrm{Bcl}-2$ is the anti-apoptosis protein, previously reported have demonstrated that apoptosis and autophagy occur simultaneously. The antitumor function of LicA was mainly through inducing apoptosis. The results have proven that LicA-induced autophagy in HCCs depend on the dose and time. ROS has negative influence on normal cells, but have dual role for cancer cells. ROS can promote tumor growth through inducing DNA damage and activating oncogenes. DeNicola et al reported that Nrf2 was in high expression state in various cancer cells, and Nrf2 could decrease the intracellular ROS generation, remove the free radicals in cells, moreover, cancer cells could not proliferate without Nrf2 expression, therefore, ROS also can inhibit cancer cell proliferation (22). ULK1/Atg13 complex assembled by RB1CC1, FIP200 and Atg101, forms a macroautophagy, and participate in the autophagy mediation through mTOR and AMPK. Our results indicated that LicA could activate the ULK1/Atg13 complex, inducing autophagy in HCCs. Furthermore, LicA also can promote the $\mathrm{O}_{2}^{-}$generation and suppress GSH generation, contribute to ROS generation in HCCs, and the antioxidant NAC can inhibit the ROS generation induced by LicA in HCCs. Previous studies have reported that tuberous sclerosis complex (TSC) is the mutational production of TSC1 or TSC2 gene, occuring in hamartomas and neurological manifestations. TSC $1 / 2$ complex can negatively regulate the mTORC1 complex, contribute to protein synthesis, cell growth and autophagy mediation (23). PRAS40 is the substrate of PKB/Akt, it can also bind to mTORC1 complex, possessing various phosphorylation sites, including Thr246 mediated by Akt, Ser183, Ser212 and Ser 221 mediated by mTORC1 complex (12). CTMP is an endogenous inhibitor of Akt, a key mediator of insulin signals, and insulin signals mediate autophagy pathway $(14,24)$. PP2A has an important role in the mediation of cell cycle, and PTEN-induced kinase 1 can increase the phosphorylation of PP2A at Y307, moreover, Bcl-2 is downstream of PP2A in PTEN-induced kinase 1 pathway, which participates in the autophagy mediation $(15,25)$. PDK1 
is upstream of Akt, and inhibits autophagy through targeting c-Src, Rubicon as a PI3KC3 subunit can communicate with Beclin 1, contribute to autophagosome suppression $(17,19)$. In this study, ROS induced by LicA can activate TSC1/2 complex, PRAS40, CTMP and PP2A signals, and inhibit PDK1 and Rubicon signals, contributing to autophagy progress triggering. Moreover, the antioxidant can inhibit ROS generation in LicA treated HCCs. Cotreatment with LicA and NAC, inhibited and promoting cell death, and enhanced LicA-induced apoptosis in HCCs. Thus, our results may provide a new direction on rational design of clinical trials, LicA and antioxidant NAC may be a good combined regimen in HCC chemotherapy.

\section{Acknowledgements}

All the authors are very thankful to Professor Hong Zhao and Wei Yang for their support.

\section{Funding}

No funding was received.

\section{Availability of data and material}

The datasets used and/or analyzed during the current study are available from the corresponding author on reasonable request.

\section{Authors' contributions}

QN, WZ, WD and DZ contributed to the conception and design of the study. QN, WZ, GW, KW and DZ developed the methodology. QN, WZ, JW and CL acquired the data. TY, WL, GW, TZ and KW analyzed and interpreted the data. QN, WZ, WD and DZ wrote, reviewed and revised the manuscript. DZ supervized the study.

\section{Ethics approval and consent to participate}

Not applicable.

\section{Consent for publication}

Not applicable.

\section{Competing interests}

The authors declare that they have no competing interests.

\section{References}

1. Siegel RL, Miller KD and Jemal A: Cancer statistics, 2015. CA Cancer J Clin 65: 5-29, 2015.

2. Ferlay J, Shin HR, Bray F, Forman D, Mathers C and Parkin DM: Estimates of worldwide burden of cancer in 2008: GLOBOCAN 2008. Int J Cancer 127: 2893-2917, 2010.

3. Torre LA, Bray F, Siegel RL, Ferlay J, Lortet-Tieulent J and Jemal A: Global cancer statistics, 2012. CA Cancer J Clin 65 87-108, 2015.

4. Yo YT, Shieh GS, Hsu KF, Wu CL and Shiau AL: Licorice and licochalcone-A induce autophagy in $\mathrm{LNCaP}$ prostate cancer cells by suppression of Bcl-2 expression and the mTOR pathway. J Agric Food Chem 57: 8266-8273, 2009.
5. Lee CK, Son SH, Park KK, Park JH, Lim SS, Kim SH and Chung WY: Licochalcone A inhibits the growth of colon carcinoma and attenuates cisplatin-induced toxicity without a loss of chemotherapeutic efficacy in mice. Basic Clin Pharmacol Toxicol 103: 48-54, 2008.

6. Rafi MM, Rosen RT, Vassil A, Ho CT, Zhang H, Ghai G, Lambert $\mathrm{G}$ and DiPaola RS: Modulation of bcl-2 and cytotoxicity by licochalcone-A, a novel estrogenic flavonoid. Anticancer Res 20: 2653-2658, 2000.

7. Kwon HS, Park JH, Kim DH, Kim YH, Park JH, Shin HK and Kim JK: Licochalcone A isolated from licorice suppresses lipopolysaccharide-stimulated inflammatory reactions in RAW264.7 cells and endotoxin shock in mice. J Mol Med (Berl) 86: 1287-1295, 2008

8. Kim YH, Shin EK, Kim DH, Lee HH, Park JH and Kim JK: Antiangiogenic effect of licochalcone A. Biochem Pharmacol 80: $1152-1159,2010$

9. Anding AL and Baehrecke EH: Autophagy in cell life and cell death. Curr Top Dev Biol 114: 67-91, 2015.

10. Sarbassov DD, Ali SM and Sabatini DM: Growing roles for the mTOR pathway. Curr Opin Cell Biol 17: 596-603, 2005.

11. Høyer-Hansen M and Jäättelä M: AMP-activated protein kinase: A universal regulator of autophagy? Autophagy 3: 381-383, 2007.

12. Kazi AA and Lang CH: PRAS40 regulates protein synthesis and cell cycle in C2C12 myoblasts. Mol Med 16: 359-371, 2010.

13. Wong CP, Seki A, Horiguchi K, Shoji T, Arai T, Nugroho AE, Hirasawa Y, Sato F, Kaneda T and Morita H: Bisleuconothine a induces autophagosome formation by interfering with AKT-mTOR signaling pathway. J Nat Prod 78: 1656-1662, 2015.

14. Park J, Li Y, Kim SH, Yang KJ, Kong G, Shrestha R, Tran Q, Park KA, Jeon J, Hur GM, et al: New players in high fat diet-induced obesity: LETM1 and CTMP. Metabolism 63: 318-327, 2014.

15. Yin X, Zhang N and Di W: Regulation of LC3-dependent protective autophagy in ovarian cancer cells by protein phosphatase 2A. Int J Gynecol Cancer 23: 630-641, 2013.

16. Gong FR, Wu MY, Shen M, Zhi Q, Xu ZK, Wang R, Wang WJ, Zong Y, Li ZL, Wu Y, et al: PP2A inhibitors arrest G2/M transition through JNK/Sp1- dependent down-regulation of CDK1 and autophagy-dependent up-regulation of p21. Oncotarget 6: 18469-18483, 2015.

17. Deng Q, Yu X, Xiao L, Hu Z, Luo X, Tao Y, Yang L, Liu X, Chen H, Ding Z, et al: Neoalbaconol induces energy depletion and multiple cell death in cancer cells by targeting PDK1-PI3-K/Akt signaling pathway. Cell Death Dis 4: e804, 2013.

18. Karlsson I, Zhou X, Thomas R, Smith AT, Bonner MY, Bakshi P, Banga AK, Bowen JP, Qabaja G, Ford SL, et al: Solenopsin A and analogs exhibit ceramide-like biological activity. Vasc Cell 7: 5, 2015 .

19. Zhong Y, Wang QJ, Li X, Yan Y, Backer JM, Chait BT, Heintz N and Yue Z: Distinct regulation of autophagic activity by Atg14L and Rubicon associated with Beclin 1-phosphatidylinositol3-kinase complex. Nat Cell Biol 11: 468-476, 2009.

20. Kim JK, Shin EK, Park JH, Kim YH and Park JH: Antitumor and antimetastatic effects of licochalcone A in mouse models. J Mol Med (Berl) 88: 829-838, 2010.

21. Park JH, Lim HJ, Lee KS, Lee S, Kwak HJ, Cha JH and Park HY: Anti-proliferative effect of licochalcone A on vascular smooth muscle cells. Biol Pharm Bull 31: 1996-2000, 2008.

22. DeNicola GM, Karreth FA, Humpton TJ, Gopinathan A, Wei C, Frese K, Mangal D, Yu KH, Yeo CJ, Calhoun ES, et al: Oncogeneinduced Nrf2 transcription promotes ROS detoxification and tumorigenesis. Nature 475: 106-109, 2011.

23. Di Nardo A, Wertz MH, Kwiatkowski E, Tsai PT, Leech JD, GreeneColozzi E, Goto J, Dilsiz P, Talos DM, Clish CB, et al: Neuronal Tsc1/2 complex controls autophagy through AMPK-dependent regulation of ULK1. Hum Mol Genet 23: 3865-3874, 2014.

24. Chen Y, Nie H, Tian L, Tong L, Deng J, Zhang Y, Dong H and Xiong L: Sevoflurane preconditioning-induced neuroprotection is associated with Akt activation via carboxy-terminal modulator protein inhibition. Br J Anaesth 114: 327-335, 2015.

25. Qi Z, Yang W, Liu Y, Cui T, Gao H, Duan C, Lu L, Zhao C, Zhao $\mathrm{H}$ and Yang H: Loss of PINK1 function decreases PP2A activity and promotes autophagy in dopaminergic cells and a murine model. Neurochem Int 59: 572-581, 2011.

This work is licensed under a Creative Commons Attribution-NonCommercial-NoDerivatives 4.0 International (CC BY-NC-ND 4.0) License. 